\title{
Gait Speed improves EuroSCORE II prediction
}

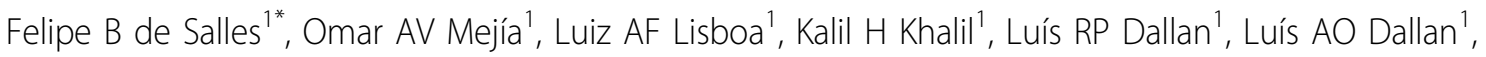 \\ Fábio B Jatene', Marco AP Oliveira², Gustavo I Judas², Sergio A de Oliveira², Orlando Petrucci ${ }^{3}$, \\ Rubens T de Barros ${ }^{4}$, Marcos G Tiveron ${ }^{4}$, Valquíria Campagnucci ${ }^{5}$, Felipe Machado ${ }^{5}$, Amauri Groppo ${ }^{6}$, \\ Rafael Tinelli ${ }^{6}$, Roberto Rocha e Silva ${ }^{7}$, Alfredo Rodrigues ${ }^{8}$, Walter Gomes ${ }^{9}$, Marcelo Nakazone ${ }^{10}$, Domingo Braile ${ }^{10}$, \\ Grupo de Estudo REPLICCAR ${ }^{11}$
}

From World Society of Cardiothoracic Surgeons 25th Anniversary Congress, Edinburgh Edinburgh, UK. 19-22 September 2015

\section{Background/Introduction}

Traditionally cardiac surgery risk scores have worse performance in elderly patients. Frailty evaluation may improve EuroSCORE II accuracy in predicting morbimortality

\section{Aims/Objectives}

Test the value of gait speed, a clinical marker for frailty, to improve the prediction of mortality and major morbidity in elderly patients undergoing cardiac surgery

\section{Method}

A multicenter prospective cohort of patients undergoing coronary artery bypass and/or valve replacement or repair from 12 tertiary care hospitals in São Paulo State/ Brazil. Patients were eligible if they were at least 60 years of age. Frailty was defined as slow gait speed, a time taken to walk $5 \mathrm{~m}$ of $\geq 6 \mathrm{~s}$. The primary end point was a composite of in-hospital post-operative mortality or major morbidity

\section{Results}

The cohort consisted of 241 patients with a mean age of $67,4 \pm 8.2$ years. One hundred and two patients $(42.3 \%)$ were classified as slow walkers before cardiac surgery. Slow gait speed patients were more likely to be female (50.9\% vs. $19.4 \%, \mathrm{p}=0.0001)$, insulin-dependence diabetic $(23.5 \%$ vs. $13.6 \%, \mathrm{p}=0.05)$, had worse EuroSCORE II $(3.9 \% \times 1.8 \%, \mathrm{p}=0.001)$. Frail patients had more prolonged length of stay $(27.5 \%$ vs $7.9 \%, \mathrm{p}=0.001)$ and more morbimortality $(32.4 \%$ vs $15.1 \%, \mathrm{p}=0.002)$. Slow gait speed was an independent predictor of the composite end point after adjusting for the EuroSCORE II (odds ratio: 2.36 ; $95 \%$ confidence interval: 1.17 to 4.76 ) and increased EuroSCORE II accuracy from $67 \%$ to $71.9 \%$ in prediction of morbimortality

\section{Discussion/Conclusion}

Impaired Gait speed patients have more morbimortality. 5 -meter gait speed test is an effective way to identify elderly patients at higher risk and is a simple way to improve EuroSCORE II performance

\section{Authors' details}

${ }^{1}$ Department of Cardiovascular Surgery, Heart Institute (InCor), School of Medicine of the University of São Paulo (USP), São Paulo, Brazil.

${ }^{2}$ Cardiovascular Surgery, Portuguese Beneficent Hospital of São Paulo, São Paulo, Brazil. ${ }^{3}$ Department of Cardiovascular Surgery, Hospital of Clinics of the University of Campinas (UNICAMP), São Paulo, Brazil. ${ }^{4}$ Department of Cardiovascular Surgery, Holy House of Mercy of Marília, Marília, Brazil.

${ }^{5}$ Department of Cardiovascular Surgery, Holy House of Mercy of São Paulo, São Paulo, Brazil. ${ }^{6}$ Department of Cardiovascular Surgery, Holy House of Mercy of Piracicaba, Piracicaba, Brazil. ${ }^{7}$ Cardiovascular Surgery, Paulo Sacramento Hospital \& SOBAM Group, Jundiaí, Brazil. ${ }^{8}$ Department of Cardiovascular Surgery, Hospital of Clinics of Ribeirão Preto, Ribeirão Preto, Brazil. 'Department of Cardiovascular Surgery, São Paulo Hospital of the Federal University of São Paulo (UNIFESP), São Paulo, Brazil. ${ }^{10}$ Department of Cardiovascular Surgery, Hospital de Base of São José do Rio Preto, São José do Rio Preto, Brazil. ${ }^{11}$ São Paulo Registry of Cardiovascular Surgery, São Paolo, Brazil.

Published: 16 December 2015

doi:10.1186/1749-8090-10-S1-A67

Cite this article as: de Salles et al:: Gait Speed improves EuroSCORE II prediction. Journal of Cardiothoracic Surgery 2015 10(Suppl 1):A67. 